\title{
The Inertial Integrated Navigation Algorithms in the Polar Region
}

\author{
Li-Jun Song $\mathbb{D}^{1},{ }^{1}$ Guang-Qiao Yang, ${ }^{1}$ Wang-Liang Zhao, ${ }^{2}$ You-Jun Ding, ${ }^{1}$ Feng Wu, \\ and Xing $\mathrm{He}^{1}$ \\ ${ }^{1} X i$ 'an University of Architecture and Technology, School of Information and Control Engineering, Xi'an, Shaanxi 710055, China \\ ${ }^{2}$ Shanghai Aerospace Control Technology Institute, Shanghai 201109, China
}

Correspondence should be addressed to Li-Jun Song; songlijun9071@sina.com

Received 24 August 2020; Revised 16 September 2020; Accepted 20 September 2020; Published 5 October 2020

Academic Editor: Tao Zhang

Copyright $\odot 2020$ Li-Jun Song et al. This is an open access article distributed under the Creative Commons Attribution License, which permits unrestricted use, distribution, and reproduction in any medium, provided the original work is properly cited.

Because the accuracy of the existing airborne navigation is lacking in the polar region, it is difficult to ensure the safety and reliability of the aircraft when it is flying over the polar region. The integrated navigation system based on the inertial navigation technology uses multi-information fusion to assist collaborative navigation and obtain an indirect grid navigation algorithm that combines the azimuth navigation algorithm and the grid navigation algorithm to solve the existing problems. This paper analyzes the principle of the inertial navigation system in the polar region, the semiphysical simulation experiments are carried out by using the navigation theory and the background engineering, and the accuracies of the integrated navigation system of the indirect grid frame in the polar region and the integrated navigation system in the middle and low latitudes are consistent, which verifies the feasibility and effectiveness of the SINS/CNS/GPS integrated navigation system in the polar region. In addition, the paper provides the theoretical basis and the application of engineering to achieve the SINS/CNS/GPS integrated navigation system in the polar region.

\section{Introduction}

The exploration, cognition, development, protection, and utilization of the polar region are a hallmark of the scientific and technological power, and there is also the inevitability of the right to speak for the development of global international science.

Since the 1990s, China has substantively participated in Arctic affairs, carried out extensive Arctic activities, and become a major active country in the Arctic. Compared with Russia, the United States, Canada, Denmark, Norway, and other countries in the near Arctic region, the geographical advantages of China in the Arctic routes and the Arctic region are not significant, but the northern territory of China is close to the polar region, the political and geographical environment of the border is complex, and there are the local disturbances constantly, so the patrol of boundary and space defense need to be strengthened. Moreover, with the status of China as a powerful country in science and technology, the increasing of military strength, and the national defense demand of China's northernmost which is a high latitude frontier, China urgently needs to increase its voice in the development of polar resources. The opening of the Arctic route has an immeasurable impact on the economic and military security of China's northeast [1-3].

The first white paper of Arctic policy, which was published by the Information Office of the State Council on January 26, 2018, points out that China is one of the countries closest to the Arctic Circle on land, and it is a "near Arctic country." It also expounds the situation and changes of the Arctic, the relationship between China and the Arctic, the objectives and basic principles of China's Arctic policy, and the main policy propositions of China's participation in Arctic affairs [4, 5].

Yang [6] points out that Russia and Canada, which are the two great Arctic powers, have the right of internal water sovereignty of the Arctic waterway in law, while other countries only have the right of innocent passage. Huang and Yun [7] suggest that our government should make full use of the status as an official observer of the Arctic Council, actively participate in the international legislation of the Arctic region, and promote the legal coordination with the 
Arctic Rim countries, so as to ensure the smooth passage of the Arctic waterway. Guo and $\mathrm{Hu}$ [8] put forward the corresponding countermeasures in the impact of the Arctic waterway on the world strategic, the opportunities, and challenges, and, in the paper, a variety of geopolitical strategies are planned for reference or implementation in dealing with the Arctic route disputes.

The paper investigates and researches the application requirements and suggestions of domestic inertial navigation system, it is agreed that the navigation in the polar region is a basic technical bottleneck for China to move from a big aviation country to a power aviation country, and it is also an important technical for opening the safe passage of polar air corridor. Based on the inertial navigation technology, the paper adopts multi-information fusion to assist in collaborative navigation, analyzes and deduces the indirect grid frame of the integrated navigation system flying over the polar region, and carries out the test of the semiphysical simulation which has the relevant background engineering to prove the support of engineering application and ensure the safety and reliability of the aircraft flying over the polar region.

\section{The Problem of Navigation in the Polar Region}

Due to high latitude, high altitude, low temperature, low air pressure, complex physical terrain, and complex climate environment, the polar region is sparsely populated. There are some problems with the reliability and safety of various navigation equipment in the polar region when there are investigation activities. The accuracy of the inertial navigation system often changes with changes of latitude and elevation, especially that a nonpolar navigation cannot meet the navigation requirements of the polar region. There are two main problems:

(1) The expression of nonpolar navigation parameters is meaningless in polar navigation. Especially in the north pole, all directions are south, which brings about trouble to polar navigation.

(2) The equipment of nonpolar navigation cannot meet the requirements of the polar navigation. Due to the magnetic field of the earth and the special astronomical conditions in the polar region, the communication and navigation of the conventional situation are very different in the middle and low latitudes, and the nonpolar navigation equipment is unable to work normally in the polar region.

In the face of an intricate navigation environment of the polar region, a safe and effective method of navigation is urgently found in the polar region. Although the inertial navigation system is considered the preferred autonomous navigation device in the polar region, the inertial navigation system has the limitation of error accumulation over time, and it is difficult to complete the highprecision, long-time cruising function only by the inertial navigation system.

\section{The Integrated Navigation System of SINS/ CNS/GPS in the Polar Region}

3.1. The Principles of SINS/CNS/GPS. The strapdown inertial navigation system (SINS) is used as a public reference system in SINS/CNS/GPS, and the global positioning system (GPS) and the celestial navigation system (CNS) are combined with SINS, respectively, to obtain the integrated navigation system of SINS/CNS/GPS. The information is processed with two independent subfilters, and then the output information of the subfilter is sent to the main filter for information fusion to obtain an optimal estimated value of the integrated error and the estimated value is to be used to correct the SINS error in real time [9-13]. The integrated navigation system in the polar region is shown in Figure 1.

3.2. The Indirect Grid Inertial Navigation. To avoid the convergence of heading reference in the polar region, the longitude is drawn parallel to the Greenwich meridian on the polar chart, and the azimuth of aircraft is also defined relative to the Greenwich meridian and its parallel lines, which is the indirect grid inertial navigation. However, the indirect grid inertial navigation has its limitations; that is, it cannot work normally near the equator. When the aircraft flies over the global, it is necessary to adopt other algorithms near the equator, so the oscillation will occur in the switching process of different navigation algorithm $[14,15]$. In order to solve the problem of oscillation, the paper proposes an indirect grid inertial navigation algorithm that combines the inertial of wandering azimuth with the grid inertial algorithm.

3.2.1. The Angle between the True North and the Grid North. An angle between the true north and the grid north is set to be $\sigma$. The grid inertial system of point $\mathrm{P}$ is the horizontal coordinate system $[16,17]$. Geographic latitude is $L$, longitude is $\lambda$, and altitude is $h$. It is rotated around a grid direction to obtain a conversion matrix between the grid inertial system $(G)$ and the geographic coordinate system $(g)$, as follows:

$$
\left[\begin{array}{l}
e_{G E} \\
e_{G N} \\
e_{G U}
\end{array}\right]=\left[\begin{array}{ccc}
\cos \sigma & -\sin \sigma & 0 \\
\sin \sigma & \cos \sigma & 0 \\
0 & 0 & 1
\end{array}\right]\left[\begin{array}{l}
e_{E} \\
e_{N} \\
e_{U}
\end{array}\right]
$$

Namely,

$$
\mathbf{C}_{g}^{G}=\left[\begin{array}{ccc}
\cos \sigma & -\sin \sigma & 0 \\
\sin \sigma & \cos \sigma & 0 \\
0 & 0 & 1
\end{array}\right] .
$$

Therefore, a transformation between the geocentric body fixed coordinate system and the grid inertial system is obtained: 


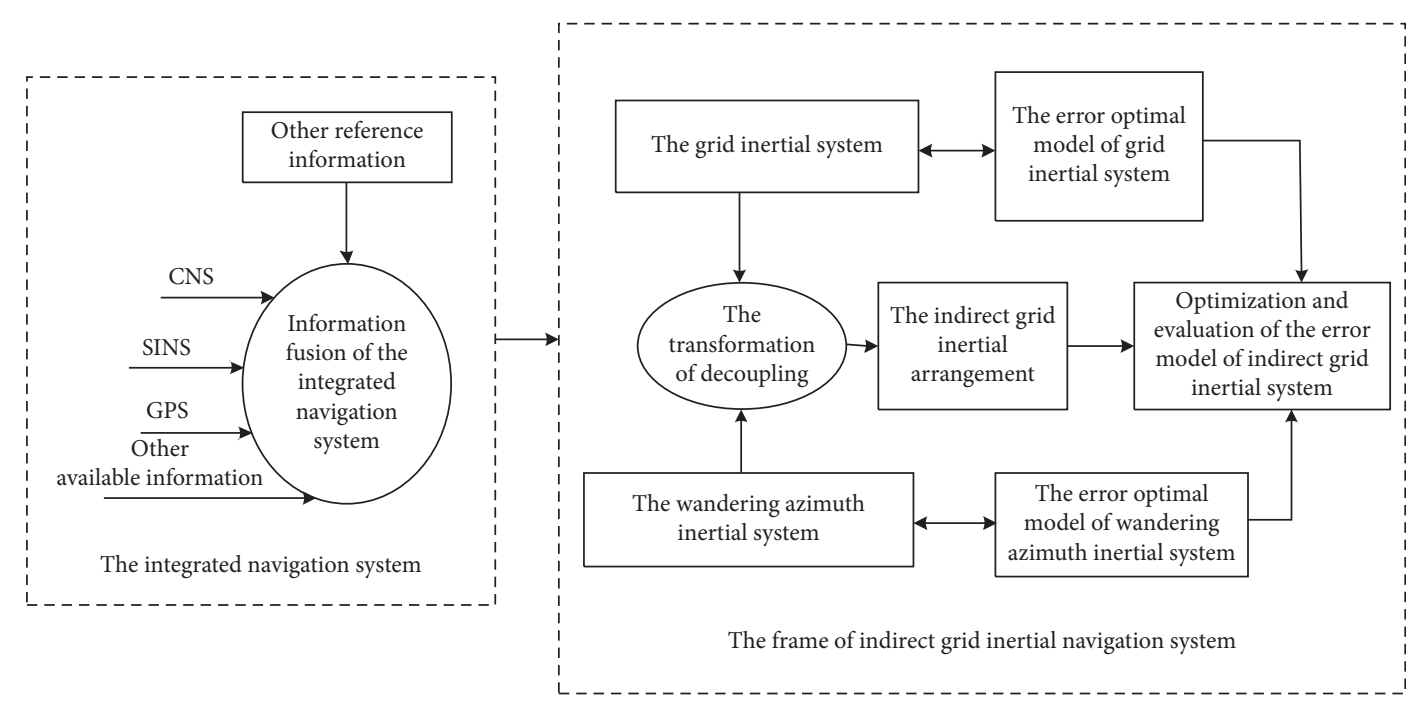

FIgURE 1: The integrated navigation system in the polar region.

$$
\begin{aligned}
{\left[\begin{array}{l}
e_{G E} \\
e_{G N} \\
e_{G U}
\end{array}\right]=} & \mathbf{C}_{g}^{G}\left[\begin{array}{l}
e_{E} \\
e_{N} \\
e_{U}
\end{array}\right]=\mathbf{C}_{g}^{G} \mathbf{C}_{e}^{g}\left[\begin{array}{l}
e_{X} \\
e_{Y} \\
e_{Z}
\end{array}\right], \\
\mathbf{C}_{e}^{G}=\mathbf{C}_{g}^{G} \mathbf{C}_{e}^{g}= & {\left[\begin{array}{ccc}
\cos \sigma & -\sin \sigma & 0 \\
\sin \sigma & \cos \sigma & 0 \\
0 & 0 & 1
\end{array}\right]\left[\begin{array}{ccc}
-\sin \lambda & \cos \lambda & 0 \\
-\sin L \cos \lambda & -\sin L \sin \lambda & \cos L \\
\cos L \cos \lambda & \cos L \sin \lambda & \sin L
\end{array}\right] } \\
= & {\left[\begin{array}{ccc}
-\sin \lambda \cos \sigma+\sin L \cos \lambda \sin \sigma & \cos \lambda \cos \sigma+\sin L \sin \lambda \sin \sigma & -\cos L \sin \sigma \\
-\sin \lambda \sin \sigma-\sin L \cos \lambda \cos \sigma & \cos \lambda \sin \sigma-\sin L \sin \lambda \cos \sigma & \cos L \cos \sigma \\
\cos L \cos \lambda & \cos L \sin \lambda & \sin L
\end{array}\right] . }
\end{aligned}
$$

The grid inertial system is recorded. Unit vectors are $\left(\mathbf{e}_{G E}, \mathbf{e}_{G N}, \mathbf{e}_{G U}\right)$. The unit vectors of the geocentric body fixed coordinate system are $\left(\mathbf{e}_{X}, \mathbf{e}_{Y}, \mathbf{e}_{Z}\right)$, with the definition of the angle between the grid north and the true north. The vectors $e_{G_{N}}$ and $e_{Y}$ are perpendicular to each other, so a relationship where an inner product is zero is satisfied:

$$
\mathbf{e}_{G N} \cdot \mathbf{e}_{Y}=0 \text {, }
$$

where $e_{G N}=\left[\begin{array}{lll}\sin \sigma & \cos \sigma & 0\end{array}\right]\left[\begin{array}{l}e_{E} \\ e_{N} \\ e_{U}\end{array}\right]$ and $e_{Y}=[\cos \lambda-$ $\sin L \sin \nmid \operatorname{os} L \sin \lambda]\left[\begin{array}{l}e_{E} \\ e_{N} \\ e_{U}\end{array}\right]$
Therefore, $\sin \sigma=(\sin L \sin \lambda / \cos \lambda) \cos \sigma$ and $\sin ^{2} \sigma+$ $\cos ^{2} \sigma=1$.

Then,

$$
\begin{aligned}
& \cos \sigma=\frac{\cos \lambda}{\sqrt{1-\sin ^{2} \lambda \cos ^{2} L}}, \\
& \sin \sigma=\frac{\sin \lambda \sin L}{\sqrt{1-\sin ^{2} \lambda \cos ^{2} L}} .
\end{aligned}
$$

$\sin \sigma$ and $\cos \sigma$ are put into $\mathbf{C}_{e}^{G}$ :

$$
C_{e}^{G}=\left[\begin{array}{ccc}
\frac{-\cos ^{2} L \sin \lambda \cos \lambda}{\sqrt{1-\sin ^{2} \lambda \cos ^{2} L}} & \sqrt{1-\sin ^{2} \lambda \cos ^{2} L} & \frac{-\sin L \cos L \sin \lambda}{\sqrt{1-\sin ^{2} \lambda \cos ^{2} L}} \\
\frac{-\sin L}{\sqrt{1-\sin ^{2} \lambda \cos ^{2} L}} & 0 & \frac{\cos L \cos \lambda}{\sqrt{1-\sin ^{2} \lambda \cos ^{2} L}} \\
\cos L \cos \lambda & \cos L \sin \lambda & \sin L \sin L
\end{array}\right] .
$$


3.2.2. The Transformation of Decoupling. In order to solve the problem of oscillation, the paper proposes an indirect grid inertial navigation algorithm that combines the inertial of wandering azimuth with the grid inertial algorithm. The azimuth and the velocity of the wandering azimuth inertial arrangement are projected to the geographic coordinate system by the relationship between the wandering coordinate system and the geographic coordinate system. The velocity and the azimuth of the wandering azimuth inertial arrangement in the polar region are projected to the grid inertial system by the relationship between the wandering azimuth inertial system and the grid inertial system to obtain the attitude information of the CNS, position information of the GPS, and velocity position information of the GPS. The projection is carried out by two independent navigation channels. One navigation channel executes the wandering azimuth inertial arrangement, outputs relevant navigation parameters in a nonpolar region, and does not output navigation information in the polar region. The other navigation channel only performs the grid inertial arrangement when the aircraft is in the polar region and performs decoupling transformation of the wandering azimuth inertial arrangement and the grid inertial arrangement. The transformation of decoupling between the wandering azimuth inertial system and the grid inertial system is shown in Figure 2.
(1) The update of attitude

$$
\dot{C}_{b}^{G}=\mathbf{C}_{b}^{G} \boldsymbol{\omega}_{G b}^{b} .
$$

The navigation coordinate system is the grid inertial system. It is assumed that $\omega_{G b}$ is the attitude rate of the aircraft from the navigation coordinate system to the body coordinate system, and the following results can be obtained:

$$
\begin{aligned}
\boldsymbol{\omega}_{G b}^{b} & =\boldsymbol{\omega}_{i b}^{b}-\boldsymbol{\omega}_{i G}^{b}=\boldsymbol{\omega}_{i b}^{b}-\mathbf{C}_{G}^{b} \boldsymbol{\omega}_{i G}^{G}=\boldsymbol{\omega}_{i b}^{b}-\mathbf{C}_{G}^{b} \boldsymbol{\omega}_{i G}^{G}, \\
\boldsymbol{\omega}_{i G}^{G} & =\boldsymbol{\omega}_{i e}^{G}+\boldsymbol{\omega}_{e G}^{G}, \\
\boldsymbol{\omega}_{i e}^{G} & =\mathbf{C}_{e}^{G} \boldsymbol{\omega}_{i e}^{e} .
\end{aligned}
$$

Put in order

$$
\boldsymbol{\omega}_{G b}^{b}=\boldsymbol{\omega}_{i b}^{b}-\mathbf{C}_{G}^{b}\left(\mathbf{C}_{e}^{G} \boldsymbol{\omega}_{i e}^{e}+\boldsymbol{\omega}_{e G}^{G}\right) .
$$

The grid inertial system of the point $P$, where a body of aircraft is located, is the horizontal coordinate system. Therefore, the horizontal component of $\omega_{e G}^{G}$ is

$$
\left[\begin{array}{c}
\omega_{e G x}^{G} \\
\omega_{e G y}^{G}
\end{array}\right]=\left[\begin{array}{cc}
\left(\frac{1}{R_{M h}}-\frac{1}{R_{N b}}\right) \sin \sigma \cos \sigma & -\left(\frac{\cos ^{2} \sigma}{R_{M b}}+\frac{\sin ^{2} \sigma}{R_{N b}}\right) \\
\frac{\sin ^{2} \sigma}{R_{M b}}+\frac{\cos ^{2} \sigma}{R_{N b}} & -\left(\frac{1}{R_{M h}}-\frac{1}{R_{N b}}\right) \sin \sigma \cos \sigma
\end{array}\right]\left[\begin{array}{c}
v_{G_{E}} \\
v_{G_{N}}
\end{array}\right]
$$

where

$$
\begin{gathered}
{\left[\begin{array}{c}
\omega_{e G x}^{G} \\
\omega_{e G y}^{G}
\end{array}\right]=\left[\begin{array}{cc}
\cos \sigma & -\sin \sigma \\
\sin \sigma & \cos \sigma
\end{array}\right]\left[\begin{array}{c}
\omega_{e g x}^{g} \\
\omega_{e g y}^{g}
\end{array}\right],} \\
{\left[\begin{array}{c}
\omega_{e g x}^{g} \\
\omega_{e g y}^{g}
\end{array}\right]=\left[\begin{array}{cc}
-\frac{1}{R_{M h}} & 0 \\
0 & \frac{1}{R_{N h}}
\end{array}\right]\left[\begin{array}{c}
v_{N} \\
v_{E}
\end{array}\right],} \\
{\left[\begin{array}{c}
v_{N} \\
v_{E}
\end{array}\right]=\left[\begin{array}{cc}
-\sin \sigma & \cos \sigma \\
\cos \sigma & \sin \sigma
\end{array}\right]\left[\begin{array}{l}
v_{G_{E}} \\
v_{G_{N}}
\end{array}\right] .}
\end{gathered}
$$

$\omega_{e G z}^{G}$ can be obtained by the definition of grid inertial system and the relationship with the geographical coordinate system.

$$
\omega_{e G z}^{G}=\omega_{e g z}^{g}-\dot{\sigma}=\frac{V_{E}}{R_{N h} \cos L}-\dot{\sigma} .
$$

It can be obtained from the equation $\cos \sigma=\left(\cos \lambda / \sqrt{1-\cos ^{2} L \sin ^{2} \lambda}\right)$. 


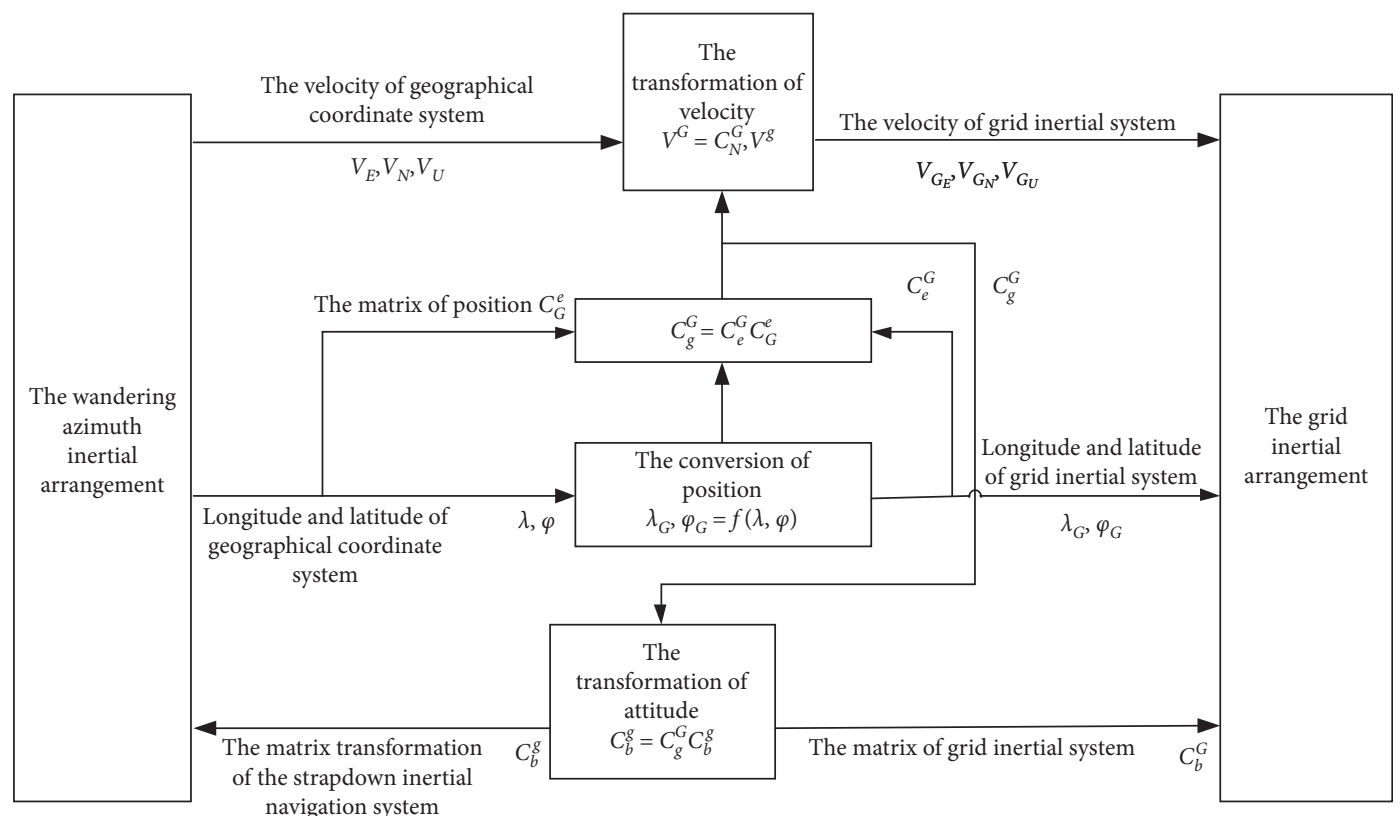

FIgURE 2: The transformation of decoupling.

$$
\begin{aligned}
\dot{\sigma} & =-\frac{1}{\sin \sigma} \cdot \frac{-\sin \lambda \cdot \dot{\lambda} \sqrt{1-\sin ^{2} \lambda \cos ^{2} L}-\cos \lambda \cdot 1 / 2\left(1-\sin ^{2} \lambda \cos ^{2} L\right)^{-1 / 2}\left[-2 \sin \lambda \cos \lambda \cos ^{2} L \cdot \dot{\lambda}+2 \sin ^{2} \lambda \sin L \cos L \cdot \dot{L}\right]}{1-\sin ^{2} \lambda \cos ^{2} L}, \\
& =\frac{\dot{\lambda}}{\sin L}+\frac{1}{\sin \sigma} \cdot \frac{\cos \sigma\left[-2 \sin \lambda \cos \lambda \cos ^{2} L \cdot \dot{\lambda}+2 \sin ^{2} \lambda \sin L \cos L \cdot \dot{L}\right]}{1-\sin ^{2} \lambda \cos ^{2} L}, \\
& =\frac{\dot{\lambda}}{\sin L}+\frac{\cos \sigma\left(\cos L \sin \sigma \cdot \dot{L}-\cos ^{2} L \cos \sigma \cdot \dot{\lambda}\right)}{\sin L}, \\
& \left\{\begin{array}{l}
\dot{L}=\frac{v_{N}}{R_{M h}}=\frac{v_{G N} \cos \sigma-v_{G E} \sin \sigma}{R_{M h}}, \\
\dot{\lambda}=\frac{v_{E}}{R_{N h} \cos L}=\frac{v_{G N} \sin \sigma+v_{G E} \cos \sigma}{R_{N h} \cos L} .
\end{array}\right.
\end{aligned}
$$

Then,

$$
\begin{aligned}
\omega_{e G z}^{G} & =\dot{\lambda} \sin L-\dot{\sigma} \\
& =\frac{\dot{\lambda}_{\sin ^{2} L-\dot{\lambda}}}{\sin L}-\frac{\cos \sigma\left(\cos L \sin \sigma \cdot \dot{L}-\cos ^{2} L \cos \sigma \cdot \dot{\lambda}\right)}{\sin L}, \\
& =-\frac{\cos ^{2} L \sin ^{2} \sigma}{\sin L} \dot{\lambda}-\frac{\cos L \sin \sigma \cos \sigma}{\sin L} \dot{L} \\
& =-\frac{\cos L \sin \sigma}{\sin L}(\cos L \sin \sigma \cdot \dot{\lambda}+\cos \sigma \cdot \dot{L}) \\
& =-\frac{\cos L \sin \sigma}{\sin L}\left[\left(\frac{\sin ^{2} \sigma}{R_{N h}}+\frac{\cos ^{2} \sigma}{R_{M h}}\right) v_{G N}+\left(\frac{1}{R_{N h}}-\frac{1}{R_{M h}}\right) \sin \sigma \cos \sigma v_{G E}\right]
\end{aligned}
$$


(2) The update of velocity

The specific force equation of the grid inertial system can be deduced by the specific force equation of the navigation system.

$$
\begin{gathered}
\dot{V}^{G}=\mathbf{f}^{G}-\left(2 \boldsymbol{\omega}_{i e}^{G}+\boldsymbol{\omega}_{e G}^{G}\right) \times \mathbf{v}^{G}+\mathbf{g}^{G}, \\
\text { where } \omega_{i e}^{G}=\mathbf{C}_{g}^{G} \omega_{i e}^{g}=\left[\begin{array}{ccc}
\cos \sigma & -\sin \sigma & 0 \\
\sin \sigma & \cos \sigma & 0 \\
0 & 0 & 1
\end{array}\right]\left[\begin{array}{c}
0 \\
\omega_{i e} \cos L \\
\omega_{i e} \sin L
\end{array}\right] \\
\text { and } \mathbf{g}^{G}=\left[\begin{array}{c}
0 \\
0 \\
-g
\end{array}\right] .
\end{gathered}
$$

\section{(3) The update of position}

The location information of longitude and latitude is obtained by integration of cosine matrix differential equation of position, and the ECEF coordinates are used to represent the real-time position of the aircraft in the high latitude.

$$
\left[\begin{array}{c}
\dot{x} \\
\dot{y} \\
\dot{z}
\end{array}\right]=C_{G}^{e} v^{G}=C_{G}^{e}\left[\begin{array}{c}
v_{G E} \\
v_{G N} \\
v_{G U}
\end{array}\right] .
$$

According to the navigation information of the polar region, if the aircraft is in the polar region, the information of the wandering azimuth inertial system is no longer output, and the polar grid navigation starts to work and outputs the navigation information. If the aircraft exits the polar region, the polar grid navigation stops working, and the information of the wandering azimuth inertial system continues to be output. The indirect grid inertial navigation can avoid the error of navigation caused by switching between the wandering azimuth navigation and the polar navigation and realize the unification of kernel and external form of navigation parameters in high latitude and low latitude.

\subsubsection{The Information Fusion of the Integrated Navigation} System in Polar Region. According to the characteristics of the integrated navigation system of SINS/GPS/CNS in the polar region, the principle of the decentralized federal filtering method is analyzed. SINS is used as a main system for output navigation parameter, and the information of CNS and GPS is used to assist in correcting various parameters of SINS to obtain a global optimal estimate value of SINS and then correct the error of the SINS in real time. The structure of no-resetting federated Kalman filter is adopted in the integrated navigation system of SINS/GPS/CNS. The design of subfilter is shown in $[18,19]$, the local optimal estimated value of public state of SINS/GPS is $\mathbf{X}_{G}$, the corresponding estimated mean square error is $P_{G}$, the local optimal estimated value of public state of SINS/CNS is XC, and the corresponding estimated mean square error is PC. Herein, according to the federal filter of global information fusion without resetting, the global optimal estimate of public state of SINS/GPS/CNS can be obtained as $\widehat{\mathbf{X}}$ and the error of estimated mean square is as follows:

$$
\left\{\begin{array}{l}
\mathbf{P}=\left(\mathbf{P}_{G}^{-1}+\mathbf{P}_{C}^{-1}\right)^{-1}, \\
\widehat{X}=\mathbf{P}\left(\mathbf{P}_{G}^{-1} \widehat{X}_{G}+\mathbf{P}_{C}^{-1} \widehat{X}_{C}\right) .
\end{array}\right.
$$

After the global optimal estimate of SINS is obtained, the error of SINS is corrected in real time, and finally the output of SINS where the error is corrected is used as the output of the integrated navigation system of SINS/GPS/CNS.

(1)The Simulation of Integrated Navigation System of SINS/GPS/CNS in the Polar Region: The state space model and the measurement model of the integrated navigation system of SINS/CNS/GPS are established with the indirect grid navigation framework. To solve the test of the integrated navigation in the polar region, a method of combining the engineering prototype and simulation is used to verify the effectiveness of the integrated navigation system of SINS/CNS/GPS based on the indirect grid frame in low and middle latitudes [20, 21]. The simulator of inertial navigation in the polar region is shown in Figure 3.

Further improvement lies in the following: the simulation test is conducted by an inertial navigation simulator of the polar region. The simulation of the SINS/GPS/CNS integrated navigation system in the polar region is set to fly for $8 \mathrm{~h}$. The calculation period of SINS is $20 \mathrm{~ms}$. The data update periods of GPS and CNS are both $1 \mathrm{~s}$.

The error of initial horizontal alignment of SINS is set to $5^{\prime}$, the error of azimuth alignment is $10^{\prime}$, the error of initial velocity is $0.1 \mathrm{~m} / \mathrm{s}$, the error of initial position is $30 \mathrm{~m}$, the drift of gyro is $0.01^{\circ} / \mathrm{h}$, and the random walk is $0.01^{\circ} / \mathrm{h}$. The drift of accelerometer is $50 \mathrm{ug}$, and the random walk is $10^{-5} \mathrm{~g} \cdot \sqrt{\mathrm{s}}$. The positioning accuracy of GPS is $25 \mathrm{~m}$, and the error of measurement velocity is $0.1 \mathrm{~m} / \mathrm{s}$. The measurement accuracy of CCD along three axes is $10^{\prime \prime}$, and the errors of installation along the three axes $x, y$, and $z$ of the aircraft are $1^{\prime}, 1^{\prime}$, and $1.5^{\prime}$. The simulation results are as follows.

It can be seen from Figure 4(f) that the installation error of the satellite tracker cannot be an estimated value when the aircraft is in static condition from 0 to $605 \mathrm{~s}$, the installation error angles of $Y$ and $Z$ directions converge rapidly when the aircraft climbed in $635 \sim 750 \mathrm{~s}$, and the installation error angles in $X$ direction converge rapidly when the aircraft have a right turn from 800 to $830 \mathrm{~s}$. Accordingly, the platform misalignment angle (Figure 4(a)) cannot be estimated in the stationary of the aircraft. In this stage, the error of misalignment angle depends on the installation error of the star tracker. When the estimation of the installation error converges, the misalignment angle also converges, and the error of convergence is less than $1^{\prime}$.

The error of velocity (Figure 4(b)) and the error of position (Figure 4(c)) in the static condition are larger, and the error of velocity and the error of position also show the form of Schuler oscillation after the convergence of misalignment angle. With the bias of gyro (Figure 4(d)) and the bias of accelerometer (Figure 4(e)) gradually estimated, the amplitude error of position and velocity error of the integrated navigation system will gradually decrease. 


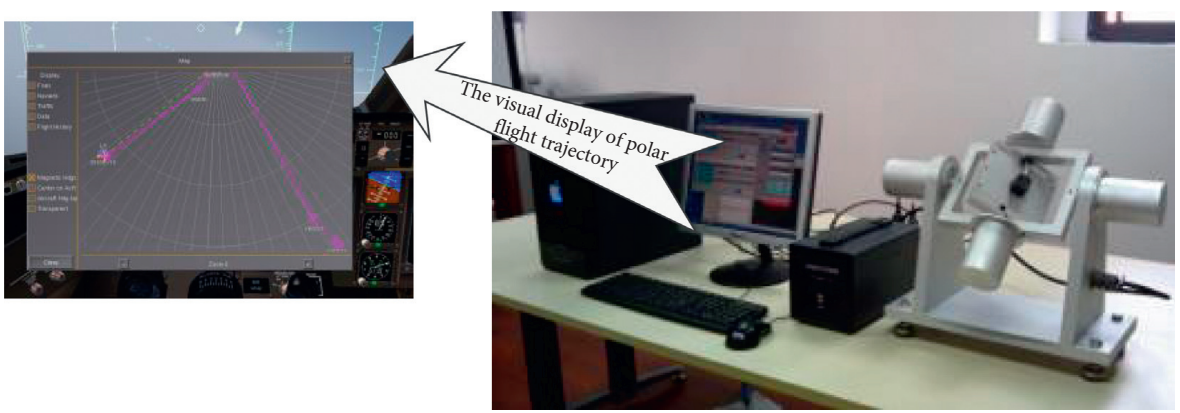

FIGURE 3: The simulator of inertial navigation in the polar region.
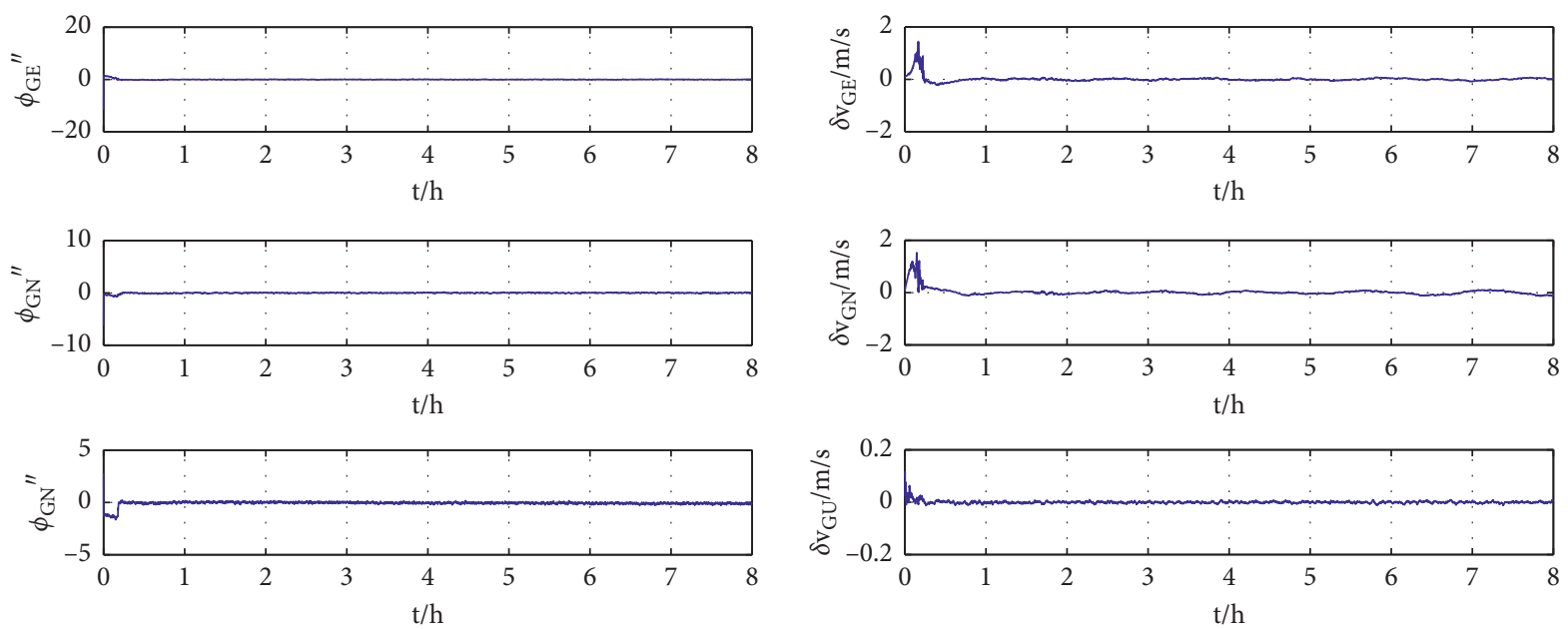

(a)

(b)
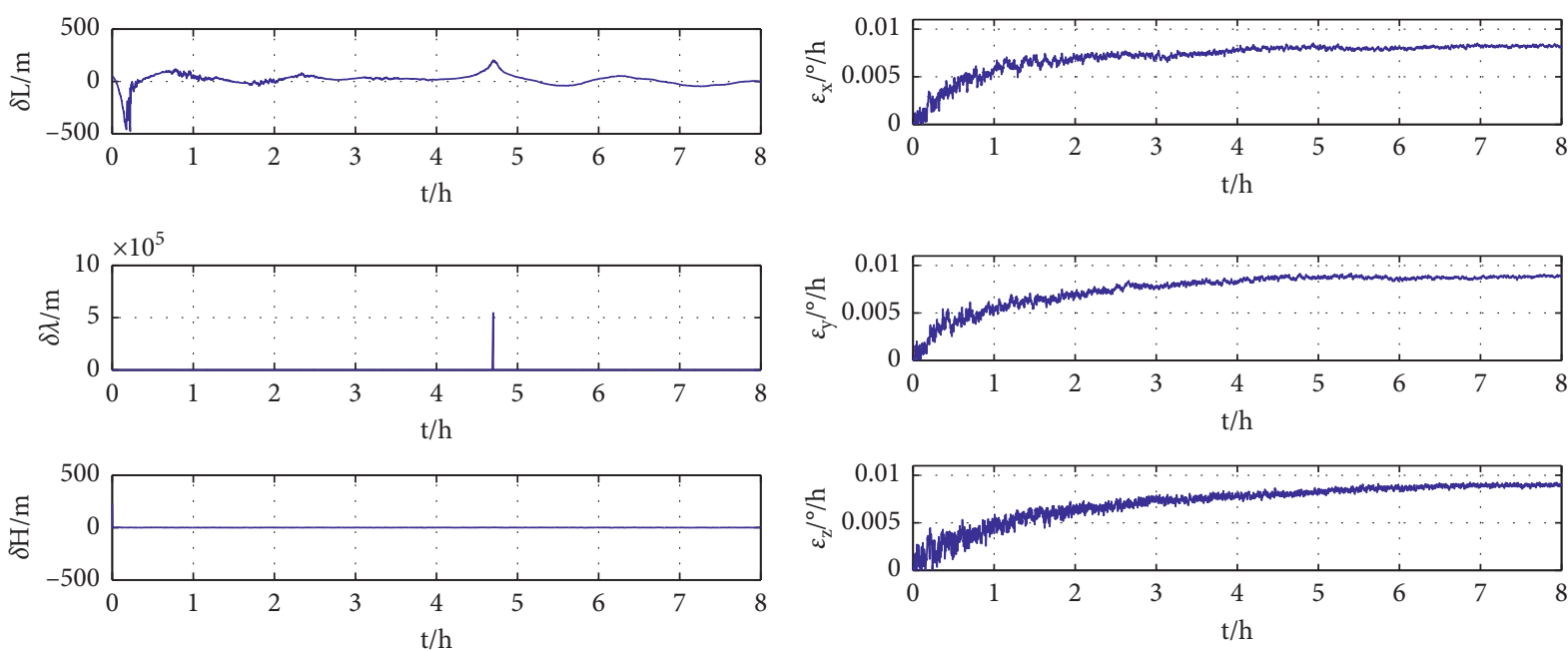

(c)

(d)

Figure 4: Continued. 

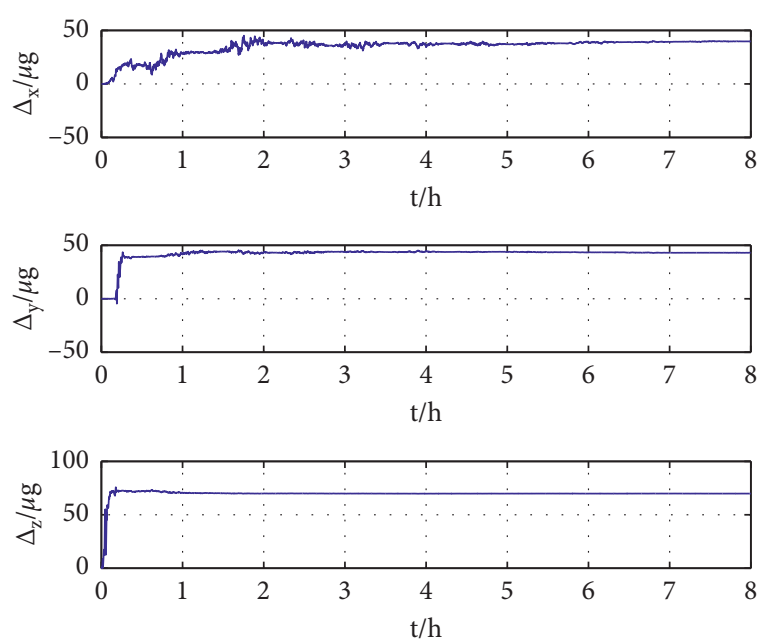

(e)
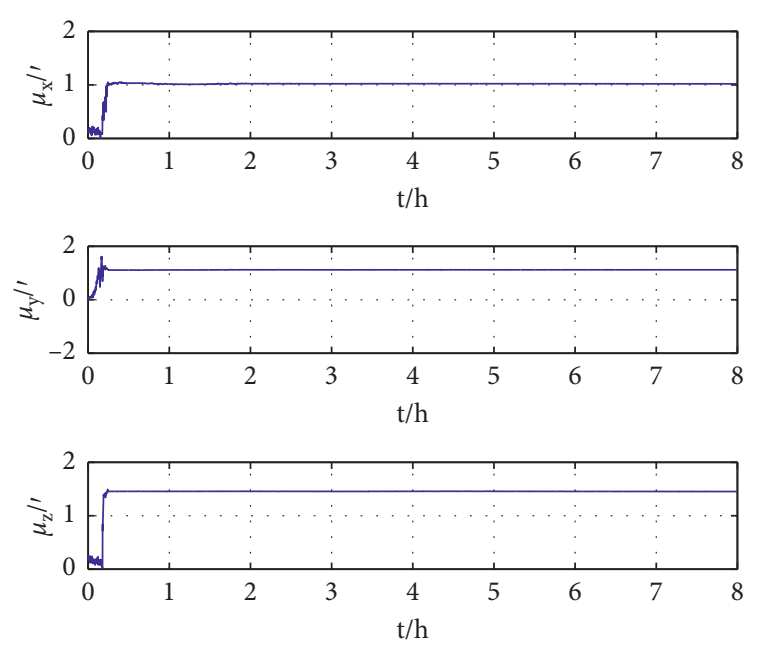

(f)

FIgURE 4: The estimation error of the navigation in the polar region. (a) The misalignment angle of the navigation in the polar region; (b) the error of velocity of the navigation in the polar region; (c) the error of position of the navigation in the polar region; (d) the drift of gyro of the navigation in the polar region; (e) the drift of accelerometer of the navigation in the polar region; (f) the installation error of CCD of the navigation in the polar region.

\section{Conclusion}

In view of the special geographical environment of "two high, two low, and three complex" in the polar region, it is difficult to locate and orient the aircraft when it is flying over the polar region, and it is easy to fail and get lost, but only the inertial navigation system cannot meet the accuracy requirements of global flight navigation. In this paper, based on the inertial navigation technology, multi-information fusion assisted cooperative navigation is used for analysis and deduction, the integrated navigation system based on the indirect grid framework is formed, and the semisimulation evaluation is carried out on the platform of engineering models. The simulation results show that the accuracy of the integrated navigation system of SINS/GPS/ CNS in polar region is consistent with that of the integrated navigation system of SINS/GPS/CNS in low latitude. The model of integrated navigation system of INS/GPS/CNS in polar region can be optimized and improved, and its navigation accuracy can be evaluated, which can provide the technology of engineering application, ensure the safety and reliability of aircraft flying over the polar region, and ensure the right of China to explore protection, development, and utilization in polar region.

\section{Data Availability}

The raw/processed data required to reach the findings in this paper cannot be shared at this time as the data also forms part of an ongoing study.

\section{Conflicts of Interest}

The authors declare that they do not have any commercial or associative interest that represents a conflict of interest in connection with the work submitted.

\section{Acknowledgments}

This work was partially supported by the Natural Science Foundation of Shaanxi Province (Project no. 2020JM-488), National Program on Key Basic Research Project (Project no. 2017YFC0704200), and the Special Scientific Research Project of the Education Department of Shaanxi Province (Project no. 20JK0728).

\section{References}

[1] X. Ma, L. I. U. Yang, and Yu Liu, "Progress and comparative analysis of domestic and international studies on Arctic shipping routes safety based on knowledge mapping," Journal of Transport Information and Safety, vol. 38, no. 223, pp. 1-12, 2020.

[2] X. Yuan and M. Hu, "Review of China's participation in the Arctic International Cooperation," Haiyang Jingji, vol. 9, no. 5, pp. 29-35, 2019.

[3] C. Zhang, "The globalization background of Arctic governance and China's participation strategy research," China Soft Science, vol. 12, no. 4, pp. 17-29, 2019.

[4] H. Wan, M. Xu, and S. Fu, "A behavior analysis of ship characteristics in Arctic Northeast Passage," Journal of Transport Information and Safety, vol. 38, no. 224, pp. 89-95, 2020.

[5] Y. Xiao, "Restraint and breakthrough: a probe into the approach of raising China's normative discourse power in the Arctic Council," Peace and Development, vol. 2020, no. 1, pp. 35-49, 2020.

[6] X. Yang, "The dispute of the Arctic passage freedom of navigation and the countermeasure of our country," Zheng Fa Lun Cong, vol. 6, no. 10, pp. 88-100, 2019.

[7] D. Huang and Y. Yun, "The embeddedness of responsibilities: the path choice of China's participation in Arctic security governance," Journal of Xiangtan University( Philosophy and Social Sciences), vol. 42, no. 2, pp. 13-19, 2018. 
[8] N. Guo and M. Hu, "Evaluation of comprehensive strength of stakeholders in the Arctic passage based on geo-economic benefits," Marine Economy, vol. 8, no. 6, pp. 3-12, 2018.

[9] G. Hu, W. Wang, Y. Zhong, B. Gao, and C. Gu, "A new direct filtering approach to INS/GNSS integration," Aerospace Science and Technology, vol. 77, pp. 755-764, 2018.

[10] B. Zhou, W. Zhao, Y. Rong, and X. Hu, "Robust adaptive Kalman filtering algorithm for integrated navigation based on MEMS-INS/GNSS," Navigation and Control, vol. 17, no. 4, pp. 14-20, 2018.

[11] W. Jiang, Y. Li, and C. Rizos, "Improved decentralized multisensor navigation system for airborne applications," GPS Solutions, vol. 22, no. 3, pp. 1-13, 2018.

[12] J. Chen, S. Yuan, J. Cheng, and X. Cao, "Research on integrated calibration based on improved federated Kalman filter," Navigation Positioning \& Timing, vol. 3, no. 6, pp. 21-25, 2016.

[13] G. Hu, B. Gao, Y. Zhong, and C. Gu, "Unscented Kalman filter with process noise covariance estimation for vehicular INS/ GPS integration system," Information Fusion, vol. 64, pp. 194-204, 2020.

[14] H. Zhang, L. Zhang, X. Wang, L. I. Lin, and Y. Zhong, "Optimization design and error analysis of strapdown inertial navigation system mechanization in polar region," Journal of Chinese Inertial Technology, vol. 23, no. 6, pp. 701-706, 2015.

[15] Y. Liu, W.-F. Tian, J.-K. Zhao, B. A. O. Qi-Lian, and Z. H. U. Shi-Qing, "Application of a new developed adaptive federated Kalman filter in tactical missile integrated navigation system," Journal of Chinese Inertial Technology, vol. 20, no. 5, pp. 577-582, 2012.

[16] X. Yao, M. Chen, Z. Zuo, and J. Wang, "Performance analysis on polar BDS PPP," Journal of Navigation and Positioning, vol. 6, no. 4, pp. 57-63, 2018.

[17] Q. Zhou, Y. Yue, X. Zhang, and Y. Tian, "Indirect grid inertial navigation mechanization for transpolar aircraft," Journal of Chinese Inertial Technology, vol. 22, no. 1, pp. 18-22, 2014.

[18] G. Zhang, G. Yan, J. Weng, and H. Yang, "N-vector inertial navigation mechanization algorithm for transpolar aircraft," Journal of Chinese Inertial Technology, vol. 25, no. 5, pp. 606-617, 2017.

[19] L. Song, Z. Duan, B. He et al., "Researched on SINS/GPS integrated navigation system based on grid reference frame in the polar region," Advances in Mechanical Engineering, vol. 9, no. 9, 2017.

[20] L. Song, W. Zhao, Y. Cheng et al., "Based on grid reference frame for SINS/CNS integrated navigation system in the polar regions," Complexity, vol. 2019, p. 8, Article ID 2164053, 2019.

[21] X. Huang, J. Liu, and Y. Wu, "An evaluation method for the head accuracy of inertial navigation system in flight test," Navigation and Control, vol. 2017, no. 4, pp. 7-12, 2017.

[22] Y. I. N. Lu, Z. Deng, D. Zhu, and E. N. Hu, "An information quality assessment method for multi-source integrated positioning system," Navigation Positioning \& Timing, vol. 2017, no. 6 , pp. 78-82, 2017. 